\title{
Reconfigurable and adaptable urban transportation systems: the platoon solution
}

\author{
Madeleine EL-ZAHER, Jean-Michel CONTET, Franck GECHTER, Pablo GRUER \\ Laboratoire Systèmes et Transports \\ Université de Technologie de Belfort-Montbéliard \\ 90010 Belfort cedex, France \\ Email: \{firstname.name\}@utbm.fr
}

\begin{abstract}
This work presents an approach to linear platoon control. Linear platoons are sets of vehicles which move while keeping a train configuration, without any mechanical coupling. Our proposal is intended for small urban vehicles, and could be the technological frame to define new urban transportation services as an alternative to individual cars, thereby contributing to the alleviation of heavy traffic conditions in downtown environments, with considerable gain in fuel consumption and better quality of air. The linear platoon control problem consists in defining the algorithms to be executed by each vehicle's embedded control, in order to maintain a correct train configuration during displacements. This involves controlling inter vehicle distance while minimizing the lateral error or, in other terms, making all vehicles to follow the same trajectory. The approach presented here bases on a virtual vehicle-to-vehicle interaction model inspired from physics. The proposed algorithms are evaluated through measurements performed on a vehicle simulation environment, capable to integrate detailed vehicle and terrain characteristics.
\end{abstract}

Keywords : Urban transportation system, intelligent vehicle, platoon control, multi-agents systems.

\section{Introduction}

Circulating personal cars reach the 15 millions per day in Paris, including its suburban area. Moreover, each individual vehicle is occupied in average by less than 1.3 person. This situation leads naturally to traffic jams, pollution, time loss, stress,... and is not specific to Paris since all big urban areas around the world are also confronted with such a problem. Despite available mass transportation systems, individual cars are intensively used despite plummeting oil price and other difficulties. This situation is linked to the fact that regular transportation systems suffer from their rigid time-tables and itinerary planning which are not quickly adaptable to specific user needs. Thus, in order to make common transportation systems more attractive for passenger, new innovative proposals have to be elaborated. Ideally, those new transportation systems should be capable of adaptation to different use profiles, from individual, on demand services to medium capacity passenger transportation services.

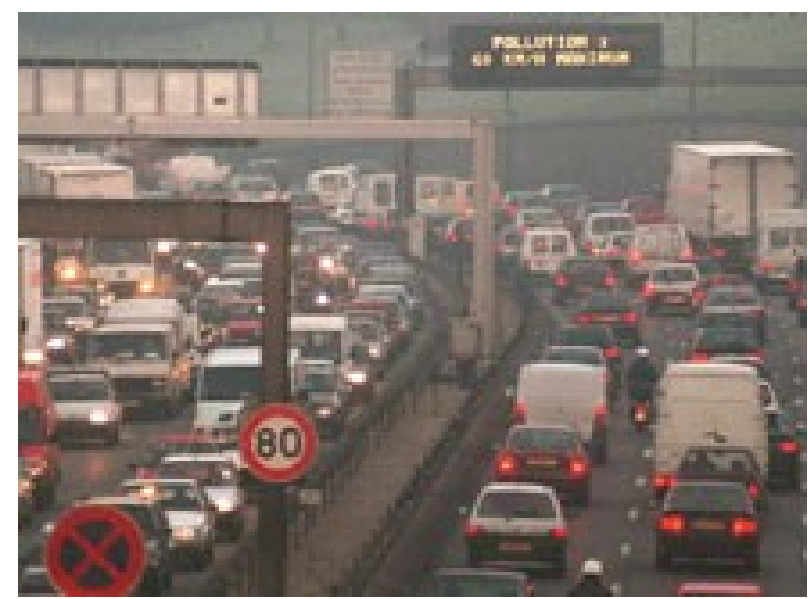

Figure 1. An example of the traffic situation in Paris during a normal day

One possible approach could base on small electrical cars, to be used either individually with small passenger capacity or integrated in variable size trains (Figure 2), for medium capacity public transportation. This kind of approach relies on vehicle units which can be used individually but are also capable of autonomy and self-organization, in order to integrate in trains of variable length. This has been particularly studied by the CRISTAL project ${ }^{1}$. The main positive points of such an approach are: (1) adaptability of system capacity depending on demand (2) possibility of individual use and (3) usage and itinerary planning in real-time, for specific demand (cultural events, problem with regular public transportation systems,...).

The key point of this innovative transportation system is the availability of a reliable virtual link between mobility units. This function called platoon function can be made thanks to several approaches depending on the sensors (laser range finder, camera,...) and the communication capacity (wifi, HF, ...) of each unit.

1. http://projet-cristal.net/ 
This paper presents an approach to linear platoon control based on a virtual mechanical coupling of vehicles, constituted by an association a spring and a damper. This paper is structured as follow: section 2 presents a state of the art of platoon solutions, section 3 describes the proposed model, section 4 draws some experimental results obtained with a urban vehicle simulator, finally, section 5 concludes the paper by giving some future works.

\section{State of art}

The linear platoon geometry control problem (cf. figure 2) has been defined as the set of functional capabilities for a vehicle to be able, on one hand, to control intervehicle distance and, on the other hand, to achieve single trajectory matching. Generally, the geometry control problem is addressed through two sub problems: longitudinal control and lateral control. Longitudinal control consists in controlling braking and acceleration in order to stabilize the distance between adjacent vehicles. Lateral control consists in determining a vehicle's direction according to intended platoon trajectory.

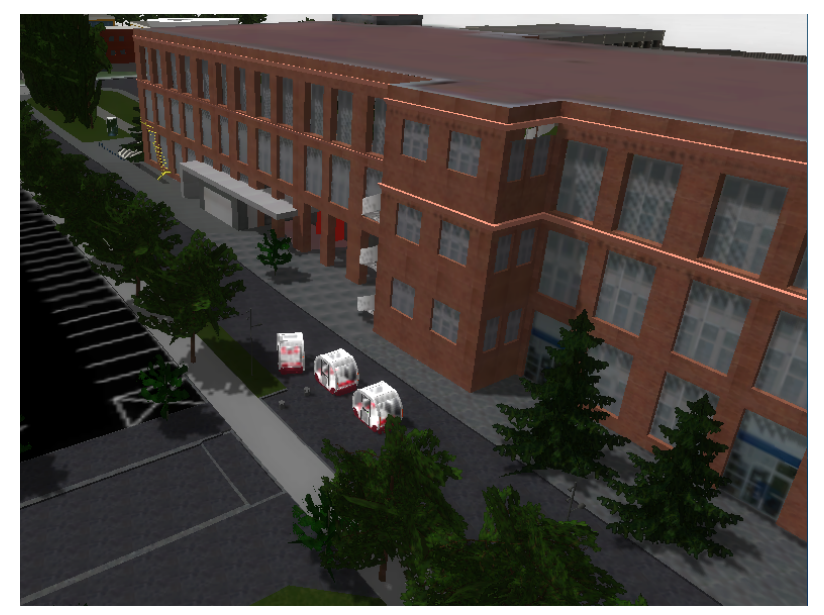

Figure 2. Simulated platoon in virtual area

Most of the approaches presented in literature can be classified as either global or local. Next paragraphs present a rapid state of the art about both of them.

Global approaches. require the presence of a decisionmaking entity, generally embedded in the head vehicle, which determines some reference information (trajectory points, steering and speed instruction, ...) and communicates them to each follower vehicles. As an example, [9], [11] uses positioning systems such as GPS RTK to compute trajectory points to be visited by follower vehicles. According to another local approach [2], the leading vehicle broadcasts driving information (steering and speed) to each follower vehicle, as in the European Chauffeur project.
Global approaches yield good trajectory matching but global positioning with GPS or other technology requires road adaptation to avoid tunneling or canyon effects. Moreover, a safe, reliable vehicle-to-vehicle communication network is required. Global control approaches yield adequate results, subject to strong constraints on sensors (high cost), road adaptation and communication reliability between vehicles.

Local approaches. perform longitudinal and lateral control based only on each vehicle's perception capabilities. Generally, vehicles are equipped with sensors which produce measurements like inter-vehicle distance vectors. Each vehicle computes its own command references (acceleration and direction) only from it's own perceptions. Most of the lateral or longitudinal control strategies proposed within local approaches use PID controllers [4], [8], [1] or other regulation-loop based algorithm [10], [7], [5]. Other proposed approaches base on a physics-inspired, inter-vehicular interaction link from which vehicle's control references can be calculated, as in [3] or in [12]. The main advantage of local approaches stems from technical simplicity of these solution (i.e. there aren't components with a specific, critical role). Moreover, local approaches require neither expensive road infrastructure, nor reliable inter-vehicle communication. They can use less expensive and more reliable sensors. On the other hand, local approaches can suffer from the anticipation error problem, which reduces the quality of trajectory matching. This matching is evaluated in terms of inter-vehicle distance error and of lateral error.

Next paragraph presents a local approach which minimizes the anticipation error by taking into account local curve properties with performance levels close to those obtained by global control.

\section{Model presentation}

A train is composed of $n$ vehicles $V_{0}, \cdots, V_{n-1}$. The first one, $V_{0}$, is assigned the navigation function. Vehicle $V_{i}(i>0)$ measures the distance vector to vehicle $V_{i-1}$ (the predecessor) and computes an interaction force according to the mechanical laws of a virtual spring damper placed between it and its predecessor. This interaction force intervenes in the computation of an acceleration command reference, to be input by the vehicle's controller.

The follower vehicle is represented by its position $\vec{V}_{i}=$ $\left[x_{i}, y_{i}\right]$. The distance between vehicles is $d=\left\|\vec{V}_{i}-\vec{V}_{i-1}\right\|$. This distance vector is calculated from the inter-vehicle distance measurement and the angle $\theta$ (cf. figure 3 ). The virtual link parameters are stiffness $k$, damping $h$, spring's resting length $l_{v}$, spring bending parameter $k_{m}$ and damper bending parameter $h_{m}$. Thus, the forces involved are : 
- Spring force $\vec{F}_{s}$ :

$$
\vec{F}_{s}=-k\left(\left\|\vec{V}_{i}-\vec{V}_{i-1}\right\|-l_{v}\right) V_{i} \vec{V}_{i-1}
$$

- Damping force $\vec{F}_{d}$ :

$$
\vec{F}_{d}=-h\left(\dot{\vec{V}}_{i}-\vec{V}_{i-1}\right)
$$

- Bending force $\vec{F}_{b}$ :

$$
\vec{F}_{b}=\overrightarrow{B A} \wedge \vec{M}
$$

From these forces, the acceleration can be computed using Newton's law of motion in the preceding vehicle reference frame :

$$
m * \vec{\gamma}=\vec{F}_{s}+\vec{F}_{d}+\vec{F}_{b}
$$

which gives:

$m * \vec{\gamma}=-k\left(\left\|\vec{V}_{i}-\vec{V}_{i-1}\right\|-l_{v}\right) V_{i} \vec{V}_{i-1}-h\left(\dot{\vec{V}}_{i}-\vec{V}_{i-1} \cdot\right)+\overrightarrow{B A} \wedge \vec{M}$

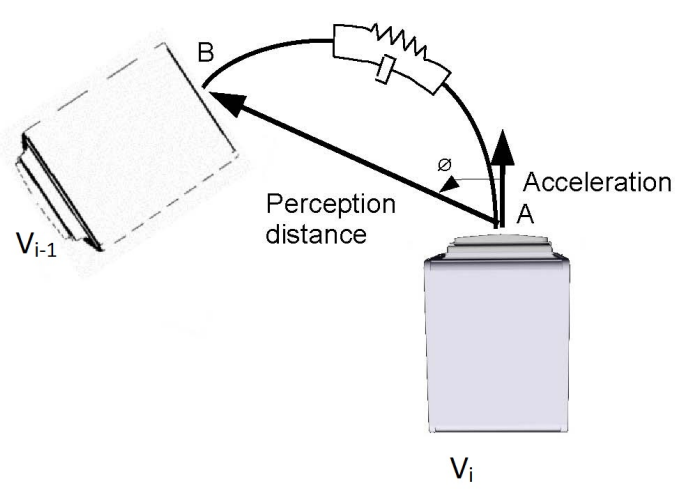

Figure 3. Virtual link between vehicles

By discrete integration, vehicle's speed, position and orientation can be calculated and the command law can be computed. In this case, it consists on vehicle's direction and speed. The choice of a command law and the of parameter values takes into account the characteristics of laboratory test vehicles. Other constraints derive from passenger transportation regulations.

\section{Simulation}

This section shows simulation results for a 3-vehicle train. Simulation were made with the VIVUS simulator [6] developed by the SeT Laboratory ${ }^{2}$. Simulations were performed on a 3D geo-localized model of Belfort's Technopole site (cf. figure 4) built from a geographical information base.

\section{2. http://set.utbm.fr/}

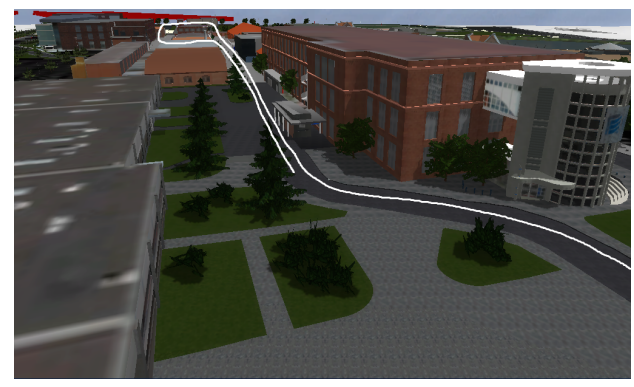

Figure 4. Experimentation path

Figure 4 shows the path used for simulations, which is representative of trajectory shapes found in urban areas (curve radius, speed profile, ...).

Simulations involved a 3-vehicle train. The leader (head vehicle) is supposed to be conducted by a human driver and each follower vehicle is equipped with the platoon control system presented in section 3 . Vehicle perception is made by a simulated laser range finder having the same characteristics (range, angle, error rate, etc.) as the real vehicle's sensor. Sensor output is composed by a distance and an angle.

\subsection{Evaluation realized using the simulator}

This subsection presents evaluations performed to assess the quality of this platoon control approach. The following cases were discussed:

- Evaluation of inter-vehicle distance: measuring distance between two successive vehicles and comparing it to the desired inter-vehicle distance.

- Evaluation of lateral deviation: measuring distance between the trajectories of a vehicle's geometric center point relatively to the same path of its predecessor. Points on the first vehicle trajectory were selected. Then, the normal straight lines by these points is drawn and the distance between the selected point and the straight line's intersection point with the predecessor's trajectory is measured.

4.1.1. Evaluation of inter-vehicle distance. Figure 5 (a) presents the case a of quick start. First vehicle accelerates to reach its maximum speed $(8 \mathrm{~km} / \mathrm{h})$. Distances between vehicles increase until an overrun of $25 \%$ compared to the desired tracking distance $(1.8 \mathrm{~m})$.

Figure 5 (b) and (c) present two cases of quick variation of speed. The first case (b) shows the distance variation resulting from a $50 \%$ speed decrease, where as second case (c) shows the same for a $75 \%$ decrease. In these two cases the distance between vehicles decreases under the desired distance and then it stabilizes after $150 \mathrm{~ms}$.

Figure (d) corresponds to a maximal acceleration in speed $(8 \mathrm{~km} / \mathrm{h})$, followed by a strong braking. As can be seen, 
distance between vehicles is stays beyond the safety distance $(0.5 \mathrm{~m})$.

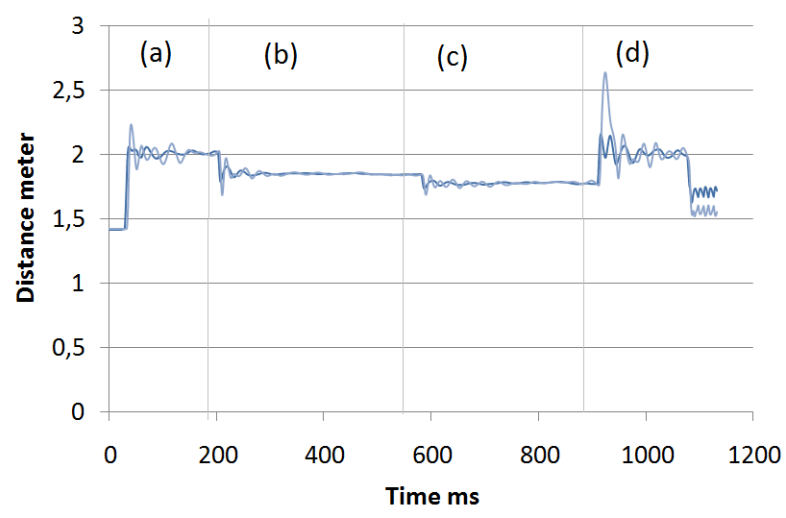

Figure 5. Inter-vehicle distance

Table 1 show inter vehicle distance variation. It is clear that despite the sharp variation of first car speed, distance between vehicles is always above the value of security distance $(0,5 \mathrm{~m})$.

\begin{tabular}{|c|c|}
\hline Critical case & Inter-vehicle distance \\
\hline \hline $\begin{array}{c}\text { Starting from 0 to } \\
\text { maximal speed }\end{array}$ & $\begin{array}{c}\text { Overrun of } 25 \% \text { compared to } \\
\text { the regular distance }\end{array}$ \\
\hline Speed variation of 50 to $75 \%$ & $\begin{array}{l}\text { Inter-vehicle distance } \\
\text { variation } 10 \text { to } 30 \%\end{array}$ \\
\hline $\begin{array}{c}\text { Safety stop from } \\
\text { maximal speed to } 0\end{array}$ & $\begin{array}{c}\text { Above the value of } \\
\text { the safety distance }\end{array}$ \\
\hline
\end{tabular}

Table 1. Evaluation of inter-vehicle distance

4.1.2. Evaluation of lateral deviation. Figure 6 shows the vehicles trajectory during a station exit. To exit the station, vehicle must turn right, then turn left. Maximal lateral error is on the point of inflection between the two bends.

This figure exhibits that maximal lateral error between first and second vehicle is around $0,5 \mathrm{~m}$, and it is around 1 $\mathrm{m}$ between first and third vehicle. Curve radius is $2,7 \mathrm{~m}$. Lateral error decrease after the inflection point to reach a value smaller than the width of a tire $(20 \mathrm{~cm})$

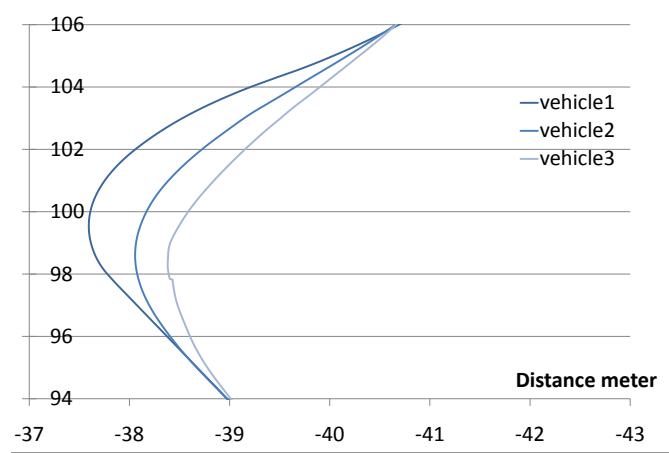

Figure 6. Simulation: lateral error during exit station

Figure 7 and table 2 allow to visualize the medium and maximal lateral error relatively to the initial wheel rotation angle and the curve radius.

\begin{tabular}{|c|c|c|c|}
\hline $\begin{array}{c}\text { Wheel } \\
\text { rotation } \\
\text { (degree) }\end{array}$ & Curve & $\begin{array}{c}\text { Medium } \\
\text { error in }\end{array}$ & $\begin{array}{c}\text { Maximal } \\
\text { error in }\end{array}$ \\
\hline 5.73 & $4 \mathrm{~m}$ & $10 \mathrm{~cm}$ & $15 \mathrm{~cm}$ \\
\hline 11.46 & $3 \mathrm{~m}$ & $20 \mathrm{~cm}$ & $25 \mathrm{~cm}$ \\
\hline 17.2 & $2,5 \mathrm{~m}$ & $50 \mathrm{~cm}$ & $60 \mathrm{~cm}$ \\
\hline 22.9 & $2 \mathrm{~m}$ & $65 \mathrm{~cm}$ & $75 \mathrm{~cm}$ \\
\hline 28.65 & $1 \mathrm{~m}$ & $70 \mathrm{~cm}$ & $1 \mathrm{~m}$ \\
\hline
\end{tabular}

Table 2. Trajectory error in curve

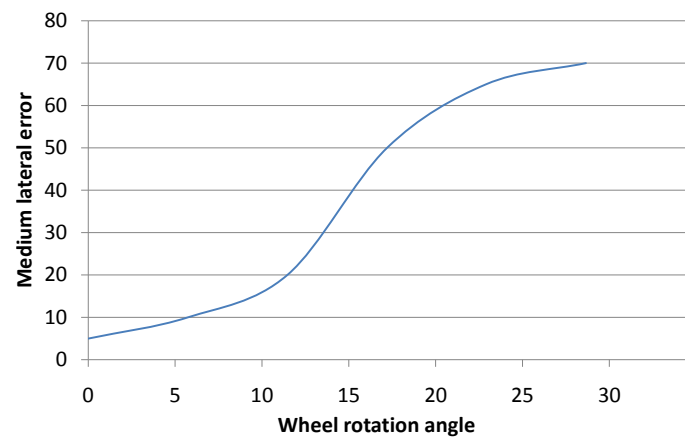

Figure 7. Simulation: lateral error in relation to the wheel rotation

Medium error decrease with wheel rotation angle decreasing. We note that in a normal running (wheel rotation $<10$ degrees), we have a lateral error smaller then $20 \mathrm{~cm}$. 


\section{Conclusion}

In this work we present an approach to the platoon control problem in the case of linear platoons. The approach bases on a virtual, physics inspired model describing interactions between a platoon vehicle and its predecessor. In this work we have put the emphasis on the principles of our approach and presented some experimental results, obtained by simulating a linear platoon. The simulator is a physics aware tool developed in our laboratory. Simulation is a previous step to material experimentation using autonomous vehicles also developed by the SeT laboratory. Future work will be devoted to finer experimentation. Among the objectives of those experiments will be to evaluate the sensitivity of the approach against the value of involved parameters. We are also investigating other physics inspired virtual interaction models, that could be adapted to platoon configurations other than trains and that could be the base for more sophisticated controls allowing, for instance, to perform dynamic vehicle insertion and ejection. Those works are done with the support of the French ANR (National Research Agency) through the ANR-VTT SafePlatoon project ${ }^{3}$ (ANR-10-VPTT-011).

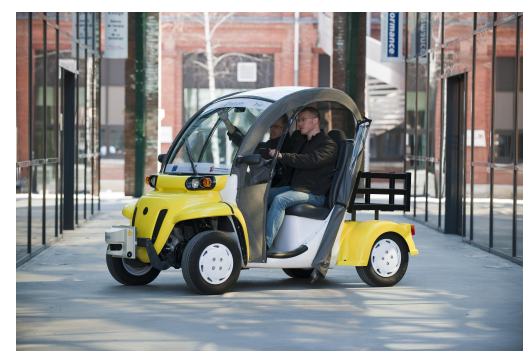

Figure 8. SeT laboratory electrical vehicle

\section{References}

[1] Pascal Daviet and Michel Parent. Longitudinal and lateral servoing of vehicles in a platoon. IEEE Intelligent Vehicles Symposium, Proceedings, pages 41 - 46, 1996. Automatic driving;Platooning techniques;

[2] Hans Fritz. Longitudinal and lateral control of heavy duty trucks for automated vehicle following in mixed traffic: Experimental results from the chauffeur project. IEEE Conference on Control Applications - Proceedings, 2:1348 - 1352, 1999. Heavy duty trucks;.

[3] S.K. Gehrig and F.J. Stein. Elastic bands to enhance vehicle following. IEEE Conference on Intelligent Transportation Systems, Proceedings, ITSC, pages 597 - 602, 2001.

[4] P. Ioannou and Z. Xu. Throttle and brake control systems for automatic vehicle following. IVHS Journal, 1(4):345 -, 1994.

3. http://web.utbm.fr/safeplatoon
[5] Nasser Kehtarnavaz, Norman C. Griswold, and Juck S. Lee. Visual control of an autonomous vehicle (bart)the vehicle-following problem. IEEE Transactions on Vehicular Technology, 40(3):654 - 662, 1991. Autonomous Vehicle;Binocular Autonomous Research Team;Visual Control;Vehicle-Following Problem;Recursive Filter;Steering Control;

[6] Oliver Lamotte, Stephane Galland, Jean-Michel Contet, and Franck Gechter. Submicroscopic and physics simulation of autonomous and intelligent vehicles in virtual reality. Advances in System Simulation, International Conference on, 0:28-33, 2010.

[7] Hyeongcheol Lee and Masayoshi Tomizuka. Adaptive vehicle traction force control for intelligent vehicle highway systems (ivhss). IEEE Transactions on Industrial Electronics, 50(1):37 $-47,2003$.

[8] John J. Moskwa and J. Karl Hedrick. Nonlinear algorithms for automotive engine control. IEEE Control Systems Magazine, 10(3):88 - 93, 1990.

[9] J. Bom P. Martinet, B. Thuilot. Autonomous navigation and platooning using a sensory memory. International IEEE Conference on Intelligent Robots and Systems, IROS'06, Beijing, China, October 2006, 2006.

[10] Shahab Sheikholeslam and Charles A. Desoer. Longitudinal control of a platoon of vehicles with no communication of lead vehicle information: A system level study. IEEE Transactions on Vehicular Technology, 42(4):546 - 554, 1993.

[11] Myung Jin Woo and Jae Weon Choi. A relative navigation system for vehicle platooning. SICE 2001. Proceedings of the 40th SICE Annual Conference. International Session Papers (IEEE Cat. No.01TH8603), pages 28 - 31, 2001.

[12] Soo-Yeong Yi and Kil-To Chong. Impedance control for a vehicle platoon system. Mechatronics (UK), 15(5):627 - 38, 2005. 